\title{
Perceived shape regularity does not depend on regularities along the contour
}

\author{
Johan Hulleman, Frans Boselie 9 \\ Nijmegen Institute for Cognition and Information, University of Nijmegen, PO Box 9104 , \\ NL-6500 HE Nijmegen, The Netherlands; e-mail: boselie@nici.kun.nl \\ Received 24 September 1998, in revised form 25 March 1999
}

\begin{abstract}
In four experiments we investigated whether human observers are able to use certain regularities in polygons when they have to indicate which polygon they perceive as the more regular in a paired comparison task. From our results we conclude that regularities that are restricted to the contour do not play a role in judgments of regularity. For instance, equilateral polygons are not considered to be more regular than entirely random polygons. Only bilaterally symmetric polygons are consistently judged as regular. However, we show that this is caused by regularities across the polygon. These results are at odds with approaches that assume that regularities along the contour play an important part in visual perception.
\end{abstract}

\section{Introduction}

Perception of regularity is a frequently recurring theme in the study of vision. Indeed, there is a wealth of research available concerning the perception of mirror symmetries (Wagemans 1995). However, there are many more kinds of regularity conceivable (eg rotational symmetry, fractals); in fact, any pattern constructed according to a rule possesses regularity.

Vision researchers are only interested in perceptually salient regularities. There are only very few theories of shape perception that make explicit statements about perceptually salient regularities. And of those few, most deal with the issue only in a rather coarse way [eg the CODON model of Richards and Hoffman (1985)]. Fortunately, however, there is a theory of shape perception-structural information theory (SIT) that is very much dedicated to the description of regularities. It makes several explicit predictions about perceptually salient regularities in polygons. SIT was introduced by Leeuwenberg $(1969,1971)$. It has been updated ever since, and its most recent version was given in van der Helm and Leeuwenberg (1996).

SIT works in the following way (see figure 1). The contour of a polygon is traced, and the sequence of edge lengths and angle sizes is translated into a sequence of symbols. Identical angle sizes or edge lengths are coded by identical symbols. This sequence of

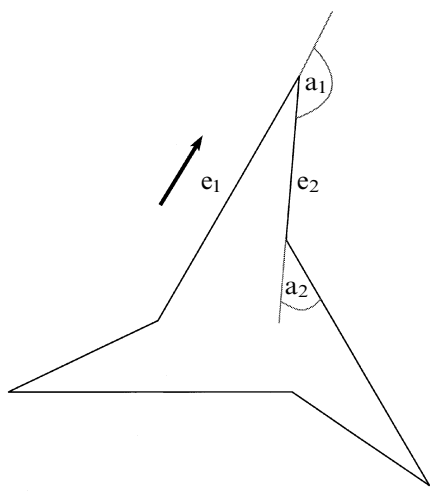

Figure 1. Translating the contour of a polygon (edges $\mathrm{e}_{i}$ and angles $\mathrm{a}_{i}$ ) into a symbol series. The resulting symbol series in this case is $e_{1} a_{1} e_{2} a_{2} e_{1} a_{1} e_{2} a_{2} e_{1} a_{1} e_{2} a_{2}$. The resulting minimal code is $3 \times\left(e_{1} a_{1} e_{2} a_{2}\right)$. Adapted from van der Helm et al (1992). 
symbols is the initial symbol series. There are three types of regularities: iterations, symmetries, and alternations (see table 1). These regularities are extracted as much as possible from the initial symbol series. The more regularities there are in this symbol series (carried by the presence of identical symbols), the less symbols there will be in the end result of the extraction process, the so-called minimum code. The minimum code determines the information load of the polygon. So, there is a direct relation between the information load of a pattern and the number of regularities contained in its code.

Table 1. The three types of regularity proposed by structural information theory (adapted from van Lier et al 1994).

\begin{tabular}{llll}
\hline Regularity & Initial symbol series & Minimum code & $I$ \\
\hline Iteration & $\mathrm{a}_{1} \mathrm{a}_{1} \mathrm{a}_{1}$ & $3 \times\left(\mathrm{a}_{1}\right)$ & 1 \\
Symmetry & $\mathrm{e}_{1} \mathrm{a}_{1} \mathrm{e}_{2} \mathrm{a}_{1} \mathrm{e}_{1}$ & $\mathrm{~S}\left[\left(\mathrm{e}_{1}\right)\left(\mathrm{a}_{1}\right),\left(\mathrm{e}_{2}\right)\right]$ & 3 \\
Alternation & $\mathrm{e}_{1} \mathrm{a}_{1} \mathrm{e}_{1} \mathrm{a}_{2}$ & $\left\langle\left(\mathrm{e}_{1}\right)\right\rangle \backslash\left\langle\left(\mathrm{a}_{1}\right)\left(\mathrm{a}_{2}\right)\right\rangle$ & 3
\end{tabular}

Recently, van der Helm and Leeuwenberg (1996) have formalised the relation between a minimum code and the perceived regularity in terms of the so-called weight of evidence. This measure is based on the number of repetitions of chunks in the minimum code, relative to the total number of elements present in the minimum code.

In van der Helm and Leeuwenberg (1996, page 437), a list is given of the phenomena to which SIT has been applied successfully: judged complexity, neon effect, embeddedness, subjective contours, pattern completion, assimilation and contrast, and figureground. In recent years, the application of SIT to figural completion has received new impetus (van Lier et al 1994).

SIT yields several explicit predictions about perceptually salient regularities in polygons. For instance, every equilateral polygon will appear very regular, compared to a 'corresponding' polygon with no edges of equal length. In fact, an equilateral polygon should look as regular as a bilaterally symmetric polygon, because both contain a regularity (alternation and symmetry, respectively), that is used to generate the minimum code. This is reflected in the identical values of the information load, and in the nearly identical values for weight of evidence (see table 2). Both information load and weight of evidence suggest that equilateral and bilaterally symmetric polygons will be perceived as distinctly more regular than random polygons (table 2). Repetition of angle sizes is another type of regularity according to SIT. It should yield polygons that look less regular than either symmetric or equilateral polygons, but look more regular than their totally random counterparts (see table 2).

Table 2. Examples of the minimum code, information load $(I)$, and weight of evidence $(W)$ for the polygon types used in experiments 1 to 4 .

\begin{tabular}{llll}
\hline Polygon type & Minimum code & $I$ & $W$ \\
\hline Symmetrical [S] & $\mathrm{S}\left[\left(\mathrm{a}_{1}\right)\left(\mathrm{e}_{1}\right)\left(\mathrm{a}_{2}\right)\left(\mathrm{e}_{2}\right) \ldots\right]$ & 17 & $\frac{1}{2}$ \\
Equilateral [E] & $\langle(\mathrm{e})\rangle \backslash\left\langle\left(\mathrm{a}_{1}\right)\left(\mathrm{a}_{2}\right) \ldots\left(\mathrm{a}_{16}\right)\right\rangle$ & 17 & $\frac{15}{32}$ \\
Equiangular [A] & $\left\langle\left(\mathrm{a}_{1}\right)\right\rangle \backslash\left\langle\left(\mathrm{e}_{1}\right)\left(\mathrm{e}_{2}\right)\right\rangle\left\langle\left(\mathrm{a}_{2}\right)\right\rangle \backslash\left\langle\left(\mathrm{e}_{3}\right)\left(\mathrm{e}_{4}\right)\left(\mathrm{e}_{5}\right)\right\rangle \ldots$ & 24 & $\frac{8}{32}$ \\
Random [R] & $\left(\mathrm{e}_{1}\right)\left(\mathrm{a}_{1}\right)\left(\mathrm{e}_{2}\right)\left(\mathrm{a}_{2}\right) \ldots\left(\mathrm{e}_{16}\right)\left(\mathrm{a}_{16}\right)$ & 32 & $\frac{0}{32}$ \\
Contour-symmetric [C] & $\mathrm{S}\left[\left(\mathrm{e}_{1}\right)\left(\mathrm{a}_{1}\right) \ldots\left(\mathrm{e}_{7}\right),\left(\mathrm{a}_{7} \mathrm{e}_{8} \mathrm{a}_{8}\right)\right] \mathrm{a}_{9} \mathrm{e}_{9} \mathrm{a}_{10}$ & 20 & $\frac{13}{32}$
\end{tabular}

Note. All types of polygons have 16 edges $\left(e_{i}\right)$ and 16 angles $\left(a_{i}\right)$. This means that the initial symbol series has an information load of 32 for all polygon types.

${ }^{a}$ The information load and weight of evidence of the A-polygons depend on the particular arrangements of concave and convex angles; the values shown here are typical values. 
There are alternatives to SIT's view that regularities along the contour, as for instance repetitions of edge lengths and angle sizes, are perceptually salient. For instance, Baylis and Driver (1995) argued that the perceptual salience of bilateral symmetry is caused by the identical description that both sides receive in terms of relative layout of component parts. Baylis and Driver suggested that these parts are defined by the minima rule of Hoffman and Richards (1984). In other words, regularities of single edge lengths and angle sizes are not enough; corresponding part descriptions on opposite sides of a polygon are responsible for high degrees of judged regularity. If this is the case, we would predict that only bilaterally symmetric polygons would be judged to be regular, and that equilateral polygons, equiangular polygons, and random polygons would be placed in a single equivalence class of polygons that are not considered regular.

In our first experiment we used four types of polygons (see figure 2 for examples). Bilaterally symmetric polygons (S-polygons), equilateral polygons [equal edges] E-polygons), equiangular polygons [a single concave and a single convex angle size] (A-polygons), and polygons with random angle sizes and random edge lengths (R-polygons).

According to SIT, this experiment will yield three equivalence classes of regular polygons. The first class contains bilaterally symmetric and equilateral polygons $\{\mathrm{S}, \mathrm{E}\}$, the second class contains the equiangular polygons $\{\mathrm{A}\}$, and the third class contains the random polygons $\{\mathbf{R}\}$. According to the part-based account, there will be only two equivalence classes: one containing the symmetric polygons $\{\mathbf{S}\}$, the other containing the other three types of polygons $\{\mathrm{E}, \mathrm{A}, \mathrm{R}\}$.

\section{Symmetric}
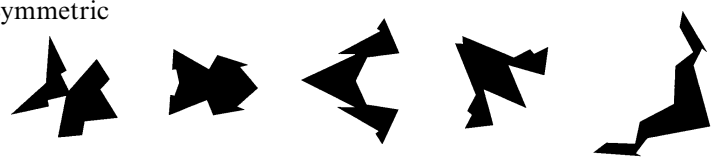

\section{Equilateral}
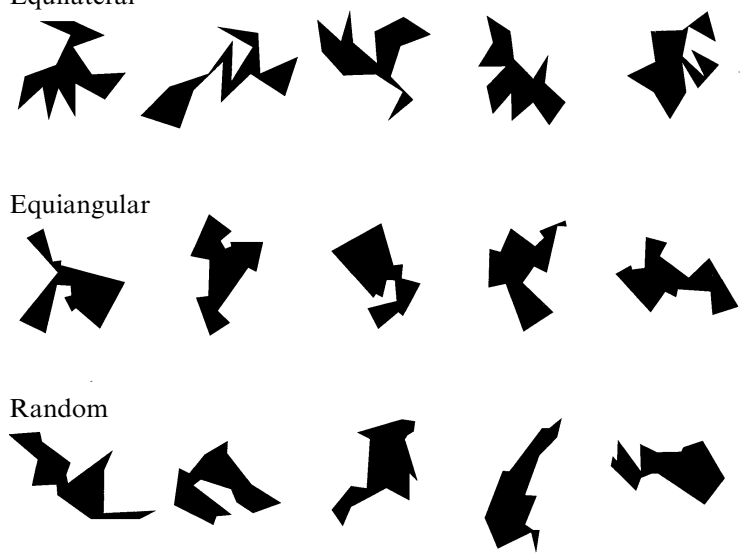

Contour-symmetric
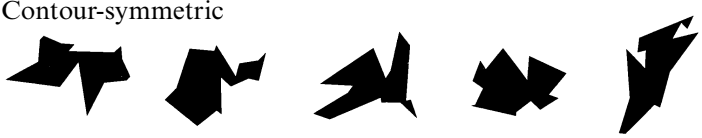

Figure 2. Examples of the stimuli used in experiments $1-4$.

In a paired comparison experiment, where these four different polygon types are used, the hypothesis that two types of polygons do not differ in perceived regularity yields three predictions. For instance, the hypothesis that symmetric (S) and equilateral (E) polygons are perceptually equally regular, the hypothesis $\mathrm{S}=\mathrm{E}$, yields the following three predictions: 
- The proportion of S-polygons judged more regular than E-polygons (notation [SE]) is equal to the proportion of E-polygons judged more regular than S-polygons ([ES]), ie $[\mathrm{SE}]=[\mathrm{ES}]$.

- The proportion of S-polygons judged more regular than A-polygons ([SA]) is equal to the proportion of E-polygons judged more regular than A-polygons ([EA]), ie $[\mathrm{SA}]=[\mathrm{EA}]$.

- The proportion of S-polygons judged more regular than R-polygons ([SR]) is equal to the proportion of E-polygons judged more regular than R-polygons ([ER]), ie $[\mathrm{SR}]=[\mathrm{ER}]$.

\section{Experiment 1}

\subsection{Method}

2.1.1 Participants. The eight subjects (four men and four women) were psychology undergraduates and graduates at the University of Nijmegen. All had normal or corrected-tonormal acuity by self-report. They received either course credit or a small payment for their participation.

2.1.2 Apparatus and materials. The experiment was conducted on a 486 PC with a 15 -inch SVGA $(800 \times 600$ pixels $)$ monitor. The shapes were presented in white on a black background. We used a total of 100 different shapes (see figure 2 for examples), 25 for each of the following four categories: (i) symmetric polygons [S-polygons], (ii) equilateral polygons [E-polygons], (iii) equiangular polygons [A-polygons], and (iv) random polygons [R-polygons]. The four categories had the following characteristics. Each S-polygon had pseudo-randomly chosen angle sizes and edge lengths; a mirror reversal generated the other half. The E-polygons had pseudo-randomly chosen angle sizes, but fixed edge lengths $(2.8 \mathrm{deg})$. The A-polygons had pseudo-randomly chosen edge lengths, but fixed angle sizes $\left(77^{\circ}\right.$ for the convex angles and $99^{\circ}$ for the concave angles). Finally, the R-polygons had pseudo-randomly chosen angle sizes and edge lengths. The minimal size difference between the pseudo-randomly chosen edge lengths (between 0.35 and $5.3 \mathrm{deg}$ ) was at least $5 \%$. This was also true for the angle sizes (chosen between $20^{\circ}$ and $160^{\circ}$ ).

All stimuli were polygons with nine convex angles and seven concave angles. The minimal extension in the horizontal direction was $5.5 \mathrm{deg}$, the maximal extension was $13.5 \mathrm{deg}$. Vertical extensions were between 5.3 and $15.1 \mathrm{deg}$. The edge lengths of the smallest circumscribing rectangle were 5.8 and $5.9 \mathrm{deg}$, those of the largest were 12 and $12.9 \mathrm{deg}$.

2.1.3 Procedure. The subjects sat in a normally lit room. Each display consisted of two shapes that had to be compared. The pair of categories was randomly chosen from the six possible pairs (symmetric-equilateral [SE], symmetric-equiangular [SA], symmetric-random [SR], equilateral-equiangular [EA], equilateral-random [ER], equiangular-random [AR]). Each individual shape was randomly chosen from the 25 shapes present in a category. The screen was split into a left and a right half, and in each of them a shape was presented. The shapes were randomly assigned to one half of the screen and centred on this half. The task of the subjects was to decide which of the two shapes presented on the screen appeared most regular. If the left shape was perceived as most regular, the subjects had to push the left button of a button box, otherwise they had to push the right button.

The subjects received a written instruction, which did not contain any formal definition of the regularities they were supposed to judge. They also performed a small test experiment, consisting of the six possible types of comparison, to acquaint themselves with the procedure. Stimulus presentation ran as follows: A fixation cross was presented for $500 \mathrm{~ms}$, followed by the display for $500 \mathrm{~ms}$. The subject responded and, 
$700 \mathrm{~ms}$ after the answer, the fixation cross reappeared and a new sequence started. There were four blocks of 100 trials each.

2.1.4 Analysis. All 400 trials were used in the analysis. For every subject, the proportions in which one polygon type was judged more regular than another were computed for the six possible comparisons. So, for every comparison, there were eight proportions available. Subsequently, we performed a total of $18 t$-tests, to test the triplets of predictions based on the six possible hypotheses of equal regularity. For every hypothesis (eg $\mathrm{S}=\mathrm{E}$ ), we performed one $t$-test that tested whether one type was preferred over the other $([\mathrm{SE}]=[\mathrm{ES}]$, which was actually tested by comparing [SE] with 0.5$)$, and two paired $t$-tests to see whether the polygon types were preferred in the same way when they were compared with the two remaining polygon types (ie $[\mathrm{SA}]=[\mathrm{EA}]$, and $[\mathrm{SR}]=[\mathrm{ER}]$ ).

\subsection{Results and discussion}

In figure 3 we see the mean proportions for the six comparisons. Between-subjects variability is fairly low (SDs are around 10\% for all the comparisons). The results of the $t$-tests are shown in table 3 . The outcome of the experiment is in total concordance with the predictions of a part-based approach of regularity, whereas the concordance with the predictions of SIT is only marginal. Only in six of the eighteen cases are the predictions of SIT supported by the data; the equilateral polygons are neither as regular as symmetric polygons, nor are they more regular than equiangular or random polygons. Furthermore, the equiangular polygons are not judged to be more regular than random ones. Apparently, there are only two equivalence classes of judged regularity for the subjects in this experiment. The first class contains symmetric polygons, the second the other three polygon types.

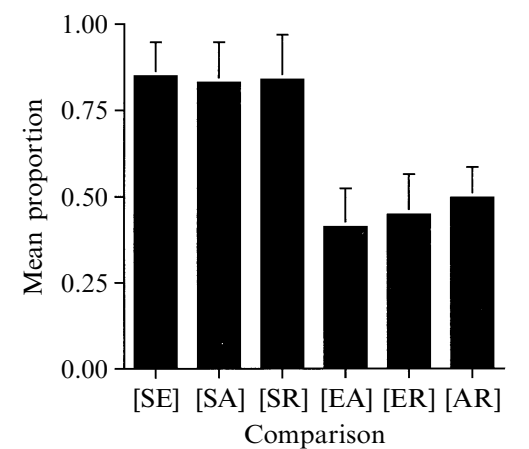

Figure 3. Experiment 1. Mean proportions of $\mathrm{X}$ judged more regular than $\mathrm{Y}$ in comparison $[\mathrm{XY}]$. For instance, in $85 \%$ of the direct comparisons, symmetric polygons were judged more regular than equilateral polygons. $\mathrm{S}$, symmetric; E, equilateral; A, equiangular; R, random. The error bars represent standard deviations. They are based on the between-subjects variability.

In accordance with the predictions of both SIT and the part-based approach, symmetric polygons are judged as more regular than equiangular and random polygons. This raises the question which of the two approaches is correct. Whereas SIT's prediction is based on the difference in the amount of regularity present along the contour, the part-based prediction is based on the larger amount of regularity across the S-polygon. The symmetric polygons used in experiment 1 contain both types of regularity. To decide between these two models, we designed a type of symmetric polygon that does contain regularity along the contour, but does not contain regularity across the polygon: the contour-symmetric polygon. This type of polygon will be used in experiment 3. But, before, we wanted to rule out an alternative explanation for the results obtained in experiment 1 - the explanation that our subjects interpreted the term regular in their instruction to mean bilaterally symmetric. This would mean that the subjects could have resorted to random behaviour when confronted with displays that did not contain a symmetric polygon, because they simply did not know what to do. To exclude this explanation, we replicated experiment 1 without symmetric polygons. 
Table 3. Results of paired $t$-tests on the proportions in experiment $1(N=8)$.

\begin{tabular}{llllll}
\hline Equivalence & $\begin{array}{l}\text { Expected } \\
\text { equalities }\end{array}$ & \multicolumn{1}{l}{$t$} & $p$ & \multicolumn{2}{l}{ Prediction } \\
\cline { 4 - 5 } $\mathrm{S}=\mathrm{E}$ & {$[\mathrm{SE}]=[\mathrm{ES}]$} & 10.02 & $0.0001^{*}$ & - & + \\
& {$[\mathrm{SR}]=[\mathrm{ER}]$} & 8.33 & $0.0001^{*}$ & - & + \\
& {$[\mathrm{SA}]=[\mathrm{EA}]$} & 9.09 & $0.0001^{*}$ & - & + \\
$\mathrm{S}=\mathrm{A}$ & {$[\mathrm{SA}]=[\mathrm{AS}]$} & 8.00 & $0.0001^{*}$ & + & + \\
& {$[\mathrm{SE}]=[\mathrm{AE}]$} & 5.00 & $0.0016^{*}$ & + & + \\
& {$[\mathrm{SR}]=[\mathrm{AR}]$} & 9.28 & $0.0001^{*}$ & + & + \\
$\mathrm{S}=\mathrm{R}$ & {$[\mathrm{SR}]=[\mathrm{RS}]$} & 7.43 & $0.0001^{*}$ & + & + \\
& {$[\mathrm{SE}]=[\mathrm{RE}]$} & 5.02 & $0.0015^{*}$ & + & + \\
& {$[\mathrm{SA}]=[\mathrm{RA}]$} & 4.84 & $0.0019^{*}$ & + & + \\
$\mathrm{E}=\mathrm{A}$ & {$[\mathrm{EA}]=[\mathrm{AE}]$} & 2.22 & 0.0618 & - & + \\
& {$[\mathrm{ES}]=[\mathrm{AS}]$} & 0.80 & 0.4494 & - & + \\
& {$[\mathrm{ER}]=[\mathrm{AR}]$} & 1.87 & 0.1034 & - & + \\
$\mathrm{E}=\mathrm{R}$ & {$[\mathrm{ER}]=[\mathrm{RE}]$} & 1.26 & 0.2487 & - & + \\
& {$[\mathrm{ES}]=[\mathrm{RS}]$} & 0.53 & 0.6116 & - & + \\
& {$[\mathrm{EA}]=[\mathrm{RA}]$} & 1.58 & 0.1571 & - & + \\
$\mathrm{A}=\mathrm{R}$ & {$[\mathrm{AR}]=[\mathrm{RA}]$} & 0.10 & 0.9265 & - & + \\
& {$[\mathrm{AS}]=[\mathrm{RS}]$} & 1.06 & 0.3227 & - & + \\
& {$[\mathrm{AE}]=[\mathrm{RE}]$} & 0.63 & 0.5476 & - & +
\end{tabular}

Note. * Significant at the $\alpha$ level of $0.0028(0.05 / 18)$. $\alpha$ level corrected for multiple comparisons. $[\mathrm{XY}], \mathrm{X}$ judged more regular than $\mathrm{Y} ;+$, result in concordance with prediction; -, result not in concordance with prediction.

Therefore, we included only three categories of polygons in our second experiment: (i) equilateral [E-polygons], (ii) equiangular [A-polygons], and (iii) random [R-polygons]. According to SIT we will find three equivalence classes of regularity, $\{\mathrm{E}\},\{\mathrm{A}\}$, and $\{\mathrm{R}\}$, with the E-polygons considered to be the most regular, and the R-polygons considered to be the least regular. According to the part-based account, there will be only a single equivalence class $\{\mathrm{E}, \mathrm{A}, \mathrm{R}\}$.

\section{Experiment 2}

\subsection{Method}

3.1.1 Participants. The eight new subjects (three men and five women) were psychology undergraduates and graduates at the University of Nijmegen. All had normal or correctedto-normal acuity by self-report. They received either course credit or a small payment for their participation.

3.1.2 Apparatus and materials. These were exactly the same as in experiment 1, except that there were now only 75 different shapes (figure 2) because we did not use the symmetric shapes. We used the same polygons as in experiment 1.

3.1.3 Procedure. This was the same as in experiment 1. Again there were 400 trials, which means that there were now more comparisons between the three polygon types than in experiment 1.

3.1.4 Analysis. The same analysis was used as in experiment 1. Of course, there were now only three possible comparisons (equilateral-random [ER], equilateral-equiangular [EA], random-equiangular [RA]). 


\subsection{Results and discussion}

From figure 4 we can see that the between-subjects variability is again low (SDs are again around $10 \%$, and even lower for the comparison between equilateral and random polygons). The results of the $t$-tests are shown in table 4 . The presence of reliable differences between equilateral polygons and the other two polygon types indicates that the subjects were able to interpret their instruction to choose the most regular polygon, even in the absence of bilaterally symmetric polygons.

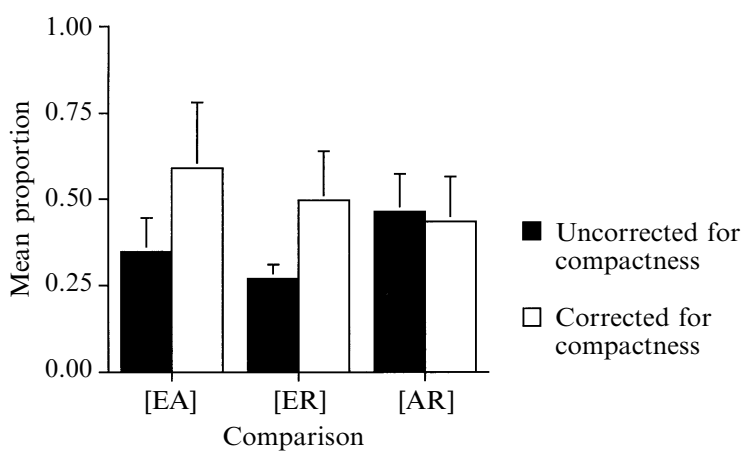

Figure 4. Experiment 2. Mean proportions of $\mathrm{X}$ judged more regular than $\mathrm{Y}$ in comparison [XY]. Black bars show the proportions before, white bars the proportions after correction for the difference in compactness. For instance, in $27 \%$ of the direct comparisons, equilateral polygons were judged more regular than random polygons. After correction for difference in compactness, this value was $50 \%$. E, equilateral; $\mathrm{A}$, equiangular; $\mathrm{R}$, random. The error bars represent standard deviations. They are based on the between-subjects variability.

Table 4. Results of paired $t$-tests on the proportions in experiment $2(N=8)$.

\begin{tabular}{|c|c|c|c|c|c|c|c|c|c|}
\hline \multirow[t]{2}{*}{ Equivalence } & \multirow{2}{*}{$\begin{array}{l}\text { Expected } \\
\text { equalities }\end{array}$} & \multirow[t]{2}{*}{$t$} & \multirow[t]{2}{*}{$p$} & \multicolumn{2}{|c|}{ Prediction } & \multicolumn{2}{|c|}{ Compactness } & \multicolumn{2}{|c|}{ Prediction } \\
\hline & & & & $\begin{array}{l}\text { SIT- } \\
\text { based }\end{array}$ & $\begin{array}{l}\text { part- } \\
\text { based }\end{array}$ & $t$ & $p$ & $\begin{array}{l}\text { SIT- } \\
\text { based }\end{array}$ & $\begin{array}{l}\text { part- } \\
\text { based }\end{array}$ \\
\hline $\mathrm{E}=\mathrm{A}$ & $\begin{array}{l}{[\mathrm{EA}]=[\mathrm{AE}]} \\
{[\mathrm{ER}]=[\mathrm{AR}]}\end{array}$ & $\begin{array}{l}4.38 \\
4.38\end{array}$ & $\begin{array}{l}0.0032 * \\
0.0032 *\end{array}$ & $\begin{array}{l}- \\
-\end{array}$ & $\begin{array}{l}- \\
-\end{array}$ & $\begin{array}{l}1.33 \\
0.69\end{array}$ & $\begin{array}{l}0.2251 \\
0.5113\end{array}$ & $\begin{array}{l}- \\
-\end{array}$ & $\begin{array}{l}+ \\
+\end{array}$ \\
\hline $\mathrm{E}=\mathrm{R}$ & $\begin{array}{l}{[\mathrm{ER}]=[\mathrm{RE}]} \\
{[\mathrm{EA}]=[\mathrm{RA}]}\end{array}$ & $\begin{array}{r}15.94 \\
7.27\end{array}$ & $\begin{array}{l}0.0001 * \\
0.0002 *\end{array}$ & $\begin{array}{l}- \\
-\end{array}$ & $\begin{array}{l}- \\
-\end{array}$ & $\begin{array}{l}0.08 \\
0.57\end{array}$ & $\begin{array}{l}0.9351 \\
0.5894\end{array}$ & $\begin{array}{l}- \\
-\end{array}$ & $\begin{array}{l}+ \\
+\end{array}$ \\
\hline $\mathrm{A}=\mathrm{R}$ & $\begin{array}{l}{[\mathrm{AR}]=[\mathrm{RA}]} \\
{[\mathrm{AE}]=[\mathrm{RE}]}\end{array}$ & $\begin{array}{l}0.94 \\
2.78\end{array}$ & $\begin{array}{l}0.3785 \\
0.0272\end{array}$ & $\begin{array}{l}- \\
-\end{array}$ & $\begin{array}{l}+ \\
+\end{array}$ & $\begin{array}{l}1.41 \\
2.03\end{array}$ & $\begin{array}{l}0.2015 \\
0.0825\end{array}$ & $\begin{array}{l}- \\
-\end{array}$ & $\begin{array}{l}+ \\
+\end{array}$ \\
\hline
\end{tabular}

Note. * Significant at the $\alpha$ level of $0.0083(0.05 / 6)$. $\alpha$ level corrected for multiple comparisons. $[\mathrm{XY}], \mathrm{X}$ judged more regular than $\mathrm{Y}$; compactness, corrected for the difference in compactness of the polygons; + , result in concordance with prediction; -, result not in concordance with prediction.

None of the predictions of SIT is supported by the data: equilateral polygons were not considered to be the most regular, nor were the equiangular polygons considered more regular than the random ones. However, the results are also in striking contradiction to the predictions of the part-based account: clearly, the three types of polygons are not seen as equally regular. These outcomes suggest that there is another factor that drives the judgments of regularity in experiment 2 .

The comments of the subjects during their debriefing suggested that this factor was the compactness of the polygons. We therefore reanalysed experiment 2 , and corrected for the difference in compactness (measured by $p^{2} / A$, the square of the perimeter divided by the area) by means of a logistic regression, where the difference in compactness between two polygons was entered as a second predictor. The results of this correction 
are shown in figure 4 and table 4 . The difference between the three polygon types disappears, and the results are now consistent with the part-based account of perceived regularity. This strongly suggests that in the absence of any clear part-based regularity (eg bilateral symmetry), compactness drives the judgments of regularity. The more compact a polygon is, the more regular it is considered to be.

The possibility that the subjects interpreted their instruction as a symmetrydetection task has been excluded in experiment 2 , because there were not any symmetric polygons present. This leads us to reject the alleged explanation of the outcome of experiment 1 -subjects did not resort to random behaviour when confronted with asymmetric polygons. In experiment 3 , we will assess the relative regularity of contoursymmetric polygons.

In constructing a contour-symmetric polygon [C-polygon] one starts from a bilaterally symmetric polygon. One of the symmetry halves of the contour receives an arbitrary rotation, followed by an arbitrary shift, after which it is reconnected to the other half (see figures 2 and 5 for examples). This means that, along the contour, these polygons retain most of the regularity of symmetric polygons. Both the weight of evidence (van der Helm and Leeuwenberg 1996), and the information load change only slightlya decrease from $\frac{1}{2}$ to $\frac{13}{32}$, and an increase from 17 to 20 , respectively (see table 2).

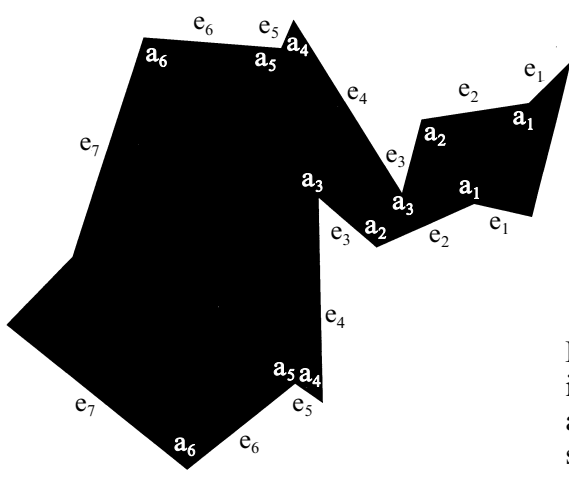

Figure 5. Example of a contour-symmetric polygon, used in experiments 3 and 4 . Identical subscripts for edges $\mathrm{e}_{i}$ and angles $\mathrm{a}_{i}$ indicate identical edge lengths and angle sizes.

So, according to SIT, the symmetric and contour-symmetric polygons will differ only slightly, or not at all, in the perceptual salience of their regularities; both types of polygons will be judged more regular than random ones. However, contour-symmetric polygons do not contain any regularity across the polygon (see figures 2 and 5). So, according to the part-based approach, symmetric and contour-symmetric polygons will differ distinctly in perceived regularity. The symmetric polygons will be judged the more regular ones, whereas the contour-symmetric polygons will not differ from random ones.

Because of the influence of compactness on the judgments, we used polygons that were closely matched in compactness in experiment 3.

\section{Experiment 3}

\subsection{Method}

4.1.1 Participants. The eight new subjects (five men and three women) were psychology undergraduates and graduates at the University of Nijmegen. All had normal or correctedto-normal acuity by self-report. They received a small payment for their participation.

4.1.2 Apparatus and materials. The experimental setup was the same as in the previous experiments. There was a total of 100 different shapes, 25 for each of the following four categories: (i) symmetric [S-polygons], (ii) contour-symmetric [C-polygons], (iii) equilateral [E-polygons], and (iv) random [R-polygons] (see figure 2 for examples). The four categories had the following characteristics: The symmetric stimuli had pseudo-randomly 
chosen angle sizes and edge lengths. The contour-symmetric stimuli were based on symmetric polygons. One of the symmetry halves of the contour was detached and rotated over a randomly chosen angle (between $20^{\circ}$ and $50^{\circ}$ ) and shifted over a random distance (between -5.3 and $5.3 \mathrm{deg}$ ) in both the horizontal and vertical direction before the sides were reconnected. The equilateral stimuli had pseudo-randomly chosen angle sizes but fixed edge lengths. Finally, the random stimuli had pseudo-randomly chosen angle sizes and edge lengths. All stimuli were polygons with nine convex angles and seven concave angles. The edge lengths and angle sizes adhered to the same restrictions as in experiments 1 and 2. The minimal extension in the horizontal direction was $5.5 \mathrm{deg}$, the maximal extension was $13.5 \mathrm{deg}$. Vertical extensions were 4.1 and $15.1 \mathrm{deg}$. The edge lengths of the smallest circumscribing rectangle were 8.1 and $4.1 \mathrm{deg}$, those of the largest were 12 and $12.9 \mathrm{deg}$.

\subsubsection{Procedure. This was the same as in experiment 1. There were again 400 trials.}

4.1.4 Analysis. The same analysis was used as in experiment 1. The six comparisons were: symmetric-equilateral [SE], symmetric-contour-symmetric [SC], symmetric-random [SR], equilateral-contour-symmetric [EC], equilateral-random [ER], and contoursymmetric-random [CR].

\subsection{Results and discussion}

From figure 6 we see that the between-subjects variability is in the same range as in the previous experiments (SDs are again around 10\%). The results of the $t$-tests are shown in table 5. Symmetric polygons are judged to be the most regular. Contoursymmetric polygons are judged to be far less regular than symmetric ones. In fact, they cannot be shown to be considered any more regular than random polygons. This result is in contradiction to SIT, but in concordance with a part-based approach. These results strongly suggest that the perceptually very salient regularity of symmetric polygons is not founded on their regularity along the contour, but rather on their regularity across the polygon, on the position that parts occupy relative to each other, as envisaged by the part-based approach.

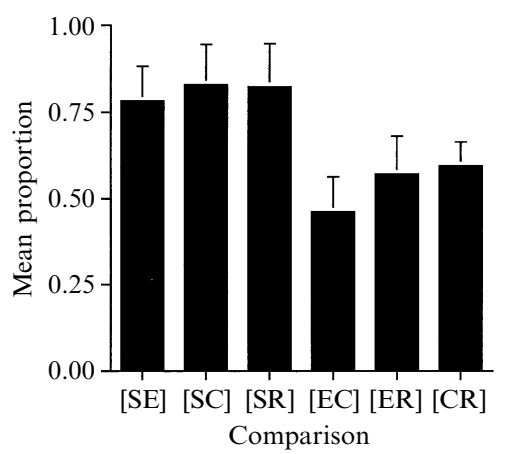

Figure 6. Experiment 3. Mean proportions of $X$ judged more regular than $\mathrm{Y}$ in comparison $[\mathrm{XY}]$. For instance, in $78 \%$ of the direct comparisons, symmetric polygons were judged more regular than equilateral polygons. $\mathrm{S}$, symmetric; $\mathrm{E}$, equilateral; $\mathrm{C}$, contour-symmetric; $\mathrm{R}$, random. The error bars represent standard deviations. They are based on the between-subjects variability.

Before we accept this conclusion, however, we have to exclude an alternative explanation for the results of experiment 3. This explanation is more in line with a sensitivity of human observers to regularities along the contour. It is possible that the subjects equated regularity with bilateral symmetry, and, as a consequence, resorted to random behaviour when confronted with a choice between a contour-symmetric polygon and another nonsymmetric polygon. In experiment 2 we saw that the absence of symmetric polygons led to a rather different pattern of preferences between the remaining three types of polygons, suggesting that the presence of symmetric polygons had a distorting effect on the pattern of preferences. Even though the largest part of the results of experiment 2 was probably caused by the difference in compactness between the polygon types, 
Table 5. Results of paired $t$-tests on the proportions in experiment $3(N=8)$.

\begin{tabular}{llllll}
\hline Equivalence & $\begin{array}{l}\text { Expected } \\
\text { equalities }\end{array}$ & $t$ & $p$ & \multicolumn{2}{l}{ Prediction } \\
\hline $\mathrm{S}=\mathrm{E}$ & {$[\mathrm{SE}]=[\mathrm{ES}]$} & 7.89 & $0.0001^{*}$ & - & + \\
& {$[\mathrm{SR}]=[\mathrm{ER}]$} & 7.84 & $0.0001^{*}$ & - & + \\
& {$[\mathrm{SC}]=[\mathrm{EC}]$} & 8.27 & $0.0001^{*}$ & - & + \\
$\mathrm{S}=\mathrm{C}$ & {$[\mathrm{SC}]=[\mathrm{CS}]$} & 7.95 & $0.0001^{*}$ & - & + \\
& {$[\mathrm{SE}]=[\mathrm{CE}]$} & 4.76 & $0.0021^{*}$ & - & + \\
& {$[\mathrm{SR}]=[\mathrm{CR}]$} & 7.34 & $0.0002^{*}$ & - & + \\
$\mathrm{S}=\mathrm{R}$ & {$[\mathrm{SR}]=[\mathrm{RS}]$} & 7.37 & $0.0002^{*}$ & + & + \\
& {$[\mathrm{SE}]=[\mathrm{RE}]$} & 5.33 & $0.0011^{*}$ & + & + \\
& {$[\mathrm{SC}]=[\mathrm{RC}]$} & 7.11 & $0.0002^{*}$ & + & + \\
$\mathrm{E}=\mathrm{C}$ & {$[\mathrm{EC}]=[\mathrm{CE}]$} & 1.08 & 0.3141 & + & + \\
& {$[\mathrm{ES}]=[\mathrm{CS}]$} & 1.55 & 0.1647 & + & + \\
& {$[\mathrm{ER}]=[\mathrm{CR}]$} & 0.85 & 0.4215 & + & + \\
$\mathrm{E}=\mathrm{R}$ & {$[\mathrm{ER}]=[\mathrm{RE}]$} & 1.82 & 0.1123 & - & + \\
& {$[\mathrm{ES}]=[\mathrm{RS}]$} & 1.68 & 0.1371 & - & + \\
& {$[\mathrm{EC}]=[\mathrm{RC}]$} & 1.27 & 0.2463 & - & + \\
$\mathrm{C}=\mathrm{R}$ & {$[\mathrm{CR}]=[\mathrm{RC}]$} & 3.83 & 0.0065 & - & + \\
& {$[\mathrm{CS}]=[\mathrm{RS}]$} & 0.46 & 0.6627 & - & + \\
& {$[\mathrm{CE}]=[\mathrm{RE}]$} & 2.79 & 0.0270 & - & +
\end{tabular}

Note. * Significant at the $\alpha$ level of $0.0028(0.05 / 18)$. $\alpha$ level corrected for multiple comparisons. $[\mathrm{XY}], \mathrm{X}$ judged more regular than $\mathrm{Y} ;+$, result in concordance with prediction; -, result not in concordance with prediction.

there is a possibility that the perfect bilateral symmetry indeed masked the contour symmetry. To settle this issue, we ran a replication of experiment 3 , without symmetric polygons. SIT predicts that the contour-symmetric polygons will be preferred over the random ones, because the contour symmetry will now no longer be eclipsed by the perfectly symmetric polygons. If, on the other hand, human observers are insensitive to regularity along the contour, we do not expect to find a preference for any polygon type. Because the polygons are now closely matched in compactness, the large preference for random polygons we observed in experiment 2 will probably not be replicated.

\section{Experiment 4}

\subsection{Method}

5.1.1 Participants. The eight new subjects (two men and six women) were psychology undergraduates at the University of Nijmegen. All had normal or corrected-to-normal acuity by self-report. They received either course credit or a small payment for their participation.

5.1.2 Apparatus and materials. These were exactly the same as in experiment 3, except that there were now only 75 different shapes (figure 2) because we did not use the symmetric shapes. We used the same polygons as in experiment 3.

5.1.3 Procedure. This was the same as in experiment 3. Again there were 400 trials, which means that there were now more comparisons between the three polygon types than in experiment 3.

5.1.4 Analysis. The same analysis was used as in experiment 3. There were now only three possible comparisons (equilateral-contour-symmetric [EC], equilateral-random [ER], and contour-symmetric-random $[\mathrm{CR}]$ ). 


\subsection{Results and discussion}

From figure 7 we see that the between-subjects variability for comparisons between equilateral and random is a little larger than in previous experiments (SD about 16\%). The results from the $t$-tests are shown in table 6 . There is not much difference between the three types of polygons, although there is a small preference for contour-symmetric polygons over random polygons. However, the preference disappears after a correction for compactness. So, there probably still is a small influence of compactness, even though the polygons were closely matched for compactness. Despite the overall matching, there remains a small difference in compactness between two individual polygons presented for a single comparison. Again, the equilateral polygons profit most from the correction for compactness. But even after the correction, neither the equilateral polygons, nor the contour-symmetric polygons are preferred above random polygons.

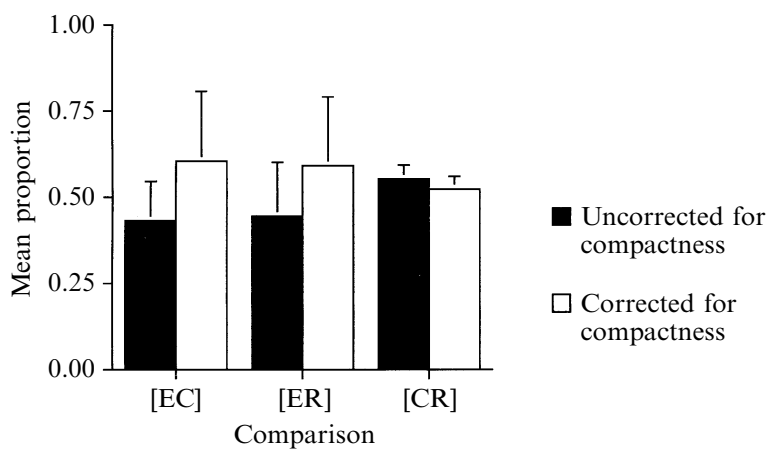

Figure 7. Experiment 4. Mean proportions of $\mathrm{X}$ judged more regular than $\mathrm{Y}$ in comparison [XY]. Black bars show the proportions before, white bars the proportions after correction for the difference in compactness. For instance, in $57 \%$ of the direct comparisons, contour-symmetric polygons were judged more regular than equilateral polygons. After correction for difference in compactness this value was $40 \%$. E, equilateral; $\mathrm{C}$, contour-symmetric; $\mathrm{R}$, random. The error bars represent standard deviations. They are based on the between-subjects variability.

Table 6. Results of paired $t$-tests on the proportions in experiment $4(N=8)$.

\begin{tabular}{|c|c|c|c|c|c|c|c|c|c|}
\hline \multirow[t]{2}{*}{ Equivalence } & \multirow{2}{*}{$\begin{array}{l}\text { Expected } \\
\text { equalities }\end{array}$} & \multirow[t]{2}{*}{$t$} & \multirow[t]{2}{*}{$p$} & \multicolumn{2}{|c|}{ Prediction } & \multicolumn{2}{|c|}{ Compactness } & \multicolumn{2}{|c|}{ Prediction } \\
\hline & & & & $\begin{array}{l}\text { SIT- } \\
\text { based }\end{array}$ & $\begin{array}{l}\text { part- } \\
\text { based }\end{array}$ & $t$ & $p$ & $\begin{array}{l}\text { SIT- } \\
\text { based }\end{array}$ & $\begin{array}{l}\text { part- } \\
\text { based }\end{array}$ \\
\hline $\mathrm{E}=\mathrm{C}$ & $\begin{array}{l}{[\mathrm{EC}]=[\mathrm{CE}]} \\
{[\mathrm{ER}]=[\mathrm{CR}]}\end{array}$ & $\begin{array}{l}1.71 \\
1.87\end{array}$ & $\begin{array}{l}0.1380 \\
0.1042\end{array}$ & $\begin{array}{l}- \\
-\end{array}$ & $\begin{array}{l}+ \\
+\end{array}$ & $\begin{array}{l}1.44 \\
0.99\end{array}$ & $\begin{array}{l}0.1943 \\
0.3570\end{array}$ & $\begin{array}{l}- \\
-\end{array}$ & $\begin{array}{l}+ \\
+\end{array}$ \\
\hline $\mathrm{E}=\mathrm{R}$ & $\begin{array}{l}{[\mathrm{ER}]=[\mathrm{RE}]} \\
{[\mathrm{EC}]=[\mathrm{RC}]}\end{array}$ & $\begin{array}{l}1.01 \\
0.43\end{array}$ & $\begin{array}{l}0.3469 \\
0.6794\end{array}$ & $\begin{array}{l}- \\
-\end{array}$ & $\begin{array}{l}+ \\
+\end{array}$ & $\begin{array}{l}1.28 \\
1.70\end{array}$ & $\begin{array}{l}0.2427 \\
0.1332\end{array}$ & $\begin{array}{l}- \\
-\end{array}$ & $\begin{array}{l}+ \\
+\end{array}$ \\
\hline $\mathrm{C}=\mathrm{R}$ & $\begin{array}{l}{[\mathrm{CR}]=[\mathrm{RC}]} \\
{[\mathrm{CE}]=[\mathrm{RE}]}\end{array}$ & $\begin{array}{l}3.79 \\
0.44\end{array}$ & $\begin{array}{l}0.0068^{*} \\
0.6723\end{array}$ & $\begin{array}{l}+ \\
-\end{array}$ & $\begin{array}{l}- \\
+\end{array}$ & $\begin{array}{l}1.71 \\
0.48\end{array}$ & $\begin{array}{l}0.1313 \\
0.6474\end{array}$ & $\begin{array}{l}- \\
-\end{array}$ & $\begin{array}{l}+ \\
+\end{array}$ \\
\hline
\end{tabular}

Note. * Significant at the $\alpha$ level of $0.0083(0.05 / 6)$. $\alpha$ level corrected for multiple comparisons. $[\mathrm{XY}], \mathrm{X}$ judged more regular than $\mathrm{Y}$; compactness, corrected for the difference in compactness of the polygons; +, result in concordance with prediction; -, result not in concordance with prediction.

In this experiment too, neither contour symmetry nor equilaterality has any effect on judgments of regularity. The judgments are primarily driven by the difference in compactness between the individual polygons. Because the results of the logistic regression analysis of the results of experiments 2 and 4 only suggest the influence of compactness on regularity judgments, we performed a separate experiment in which the subjects of experiment 4 performed paired comparisons between compact equilateral 
polygons and dispersed equilateral polygons. All of these polygons were quite comparable to the equilateral polygons used in the previous experiments. The compact polygons had a $p^{2} / A$ of approximately $50 \pm 3.5($ mean $\pm \mathrm{SD})$; the dispersed polygons had a $p^{2} / A$ of approximately $100 \pm 7.5$.

There were two conditions. In the first condition the perimeter of the compact and dispersed polygons was identical, whereas in the second condition the area was held constant. In both conditions there was a very large preference for the compact equilateral polygons $(93.4 \% \pm 7.4 \%$ and $92.8 \% \pm 7.9 \%$ for constant perimeter and constant area, respectively; mean $\pm \mathrm{SD} ; 400$ trials per subject). This shows that, in the absence of any obvious part-based regularity, the difference in compactness decides which of two polygons is considered to be more regular. This result also demonstrates that the subjects of experiment 4 were able to interpret their instruction to choose the most regular of the two polygons in a consistent manner, and it reinforces the conclusion from experiment 4 that neither contour symmetry nor equilaterality has any influence on judgments of regularity.

\section{General discussion}

We conclude that subjects do not use regularities that are restricted to the contour in their judgments of regularity. The repetition of a single edge length along the entire contour of a polygon is not enough to give the polygon an advantage over a totally random polygon. Even when we repeat long sequences of edges and angles in a mirrorsymmetric fashion, as in the contour-symmetric polygons, performance does not change.

We agree with Baylis and Driver (1995) that both the shape of the parts of the polygon, and the position these parts occupy relative to each other, on opposite sides of the polygon, are important. Parts made up of edges and angles and the area they enclose seem to be the level at which regularity is judged. If there is a repetition of parts, a polygon is considered to be regular. This view is supported by the results of experiments 1 and 2. The parts of the equilateral polygons do not show any regularity because they are all different and consequently there is no repetition of parts at all. Neither is there any repetition of parts in either the random polygons or the equiangular polygons. In the symmetric polygons, however, there is a repetition of parts, on opposite sides of the polygon, and from this stems their regularity.

But repetition of parts is not the whole story; there also has to be regularity in the positions occupied by identical parts. If there is not any regularity in their positions, there is a chance that the parts that are repeated are not formed at all, despite the fact they are present in the polygon. This is illustrated by the case of the contoursymmetric polygons in experiments 3 and 4 . Here, there are parts that could support regularity detection: pieces of contour that would receive the same part description, by pairing neighbouring negative curvature minima. However, this regularity is not perceived. An explanation was given by Kurbat (1994). He argued that human observers, owing to the hierarchical nature of many natural objects, will frequently pair nonneighbouring negative curvature minima on opposite sides of an object. In the case of contour-symmetric polygons, this often leads to parts that do not support regularity detection, because the concavities that are paired are not the ones nearest to each other on the contour, but rather the ones nearest to each other on opposite sides of the polygons. The importance of the distance between opposite sides for part segmentation has earlier been emphasised by Siddiqi and Kimia (1995). They contrasted pairing concavities nearest to each other on the contour ('limbs') with pairing concavities opposite to each other at loci where the shape is at its thinnest ('necks').

A part-based account of regularity is not enough, however. In the absence of regularity of parts in any of the polygon types, as for instance in experiments 2 and 4, it seems that human observers use another type of regularity: absence of jaggedness. 
This is reflected in the way a correction for the difference in compactness changed the preferences in experiments 2 and 4. That there is a strong perceptual role for compactness is attested in recent work by Wagemans et al (1997) on perceptual matching of perspectively versus more generally transformed polygons. They also needed a correction for compactness, because subjects used it to determine their choice. Moreover, in explicit tests, compactness correctly predicted which of two equilateral polygons was preferred in about $93 \%$ of the cases.

The value of $p^{2} / A$ reflects the presence or absence of salient acute angles. The presence of many obtuse angles in a polygon results in low values of $p^{2} / A$, whereas many salient acute angles result in high values. The equilateral polygons, in particular, have on average many salient acute angles. This is caused by the fact that the salience of acute angles is influenced by the length of the two edges forming the angle. Very acute angles are not very salient when they are formed by two small edges or by edges that differ greatly in length. In the other polygon types these inconspicuous acute angles are sometimes present. But in the equilateral polygons all the edges have a single size, and therefore any acute angle will be salient.

Our results have rather serious consequences for SIT. Although it could be argued that SIT was merely designed to yield an information load for a polygon, regularities are at the very heart of SIT, because they determine the amount of reduction in information load that is achievable when the initial symbol series is transformed into the minimum code. Moreover, the goodness measure developed within SIT, recently introduced by van der Helm and Leeuwenberg (1996), also predicts a high amount of perceived regularity in the equilateral and contour-symmetric polygons. The weight of evidence of bilaterally symmetric, equilateral, and contour-symmetric polygons is nearly equal-larger than the weight of evidence of A-polygons, and much larger than the weight of evidence in random polygons (see table 2).

It seems therefore that the use of edge lengths and angle sizes to obtain the basic codes for polygons (eg van Lier et al 1994) is not in any way equivalent to the way human observers represent polygons. This conclusion seems at odds with the large amount of literature in which SIT has used edge lengths and angle sizes to successfully describe and predict performance in visual perception tasks. The key to this apparent paradox lies in the difference between the domain in which SIT has been applied thus far and the domain which we used in our experiments. The domain of SIT thus far has consisted almost entirely of very regular polygons. Most of the time, the polygons possess at least one axis of bilateral symmetry. As we have seen, this kind of regularity is detected very easily by human subjects and it is also coded by SIT. However, this is merely a coincidence. Bilateral symmetric polygons possess several kinds of regularity. They contain regularity along the contour as well as regularity across the symmetry axis. For instance, a square is both rotationally and bilaterally symmetric. The first type of regularity is used by SIT to describe the regularity of a square, but from our results it seems that it is the second type of regularity that is used by human observers. When the two types of regularity are uncoupled, as they were in our contoursymmetric stimuli, the perceived regularity of the polygons decreases dramatically, whereas this change is hardly reflected in the minimum code. The equilateral polygons are another example. Along the contour they possess a very strong kind of regularity. But the subjects are unable to access this regularity, because it is not reflected in the relative position of corresponding part descriptions on opposite sides of the polygon.

In a way, the lack of success of SIT predicting the outcomes is not surprising. When the alternation rule is applied at the level of individual edges and angles, as it has been in our experiments, it results in a separation of angles from edges. This amounts to the destruction of the two-dimensional character of a polygon, since the parts of a polygon consist of both edges and angles. So, one bottom line of this 
discussion is that SIT should take as the minimal elements on which to apply its rules (i) an aggregate of two edges and an angle, and (ii) a single concave angle, as the connection between two aggregates. This would, for instance, make impossible the awkward coding of a square as a rotationally symmetric object, since this is only possible by applying the iteration rule to an aggregate of a single edge and a single angle. Moreover, it would also be the end of the prediction that equilateral polygons, used in these experiments, should be perceived as regular, because the alternation rule would no longer be applicable. Neither would there be any iteration of aggregates. Because every angle of the equilateral polygons we used has a different size, there is not any repetition. However, the results obtained with the contour-symmetric polygons show that there is more to be done. The contour-symmetric polygons show that the real problem with SIT lies in the fact that it does not take into account the relative positions of the aggregates on which its rules are applied. So the relative position of the aggregates should somehow be incorporated as well.

In our view, an approach like the one proposed by Baylis and Driver (1995) shows more potential of eventually guiding us to an answer to the question which types of regularity are perceived, even though it is clear that there remains a lot of experimental work to be done.

Acknowledgements. The Netherlands Organization for Scientific Research (NWO) is gratefully acknowledged for funding this project. This research was conducted while Johan Hulleman was supported by a grant of the Foundation for Behavioural Sciences of this organization (575-62-062). Parts of this research have been presented at the ECVP 1996, Strasbourg, France.

\section{References}

Baylis G C, Driver J, 1995 "Obligatory edge assignment in vision: the role of figure and part segmentation in symmetry detection" Journal of Experimental Psychology: Human Perception and Performance $211323-1342$

Helm P A van der, Leeuwenberg E L J, 1996 "Goodness of visual regularities: a non-transformational approach" Psychological Review $103429-456$

Helm P A van der, Lier R J van, Leeuwenberg E L J, 1992 "Serial pattern complexity: irregularity and hierarchy" Perception $21517-544$

Hoffman D D, Richards W, 1984 "Parts of recognition" Cognition 18 65-96

Kurbat M A, 1994 "Structural description theories: is RBC/JIM a general-purpose theory of human entry-level object recognition?" Perception 23 1339-1368

Leeuwenberg E L J, 1969 "Quantitative specification of information in sequential patterns" Psychological Review $76216-220$

Leeuwenberg E L J, 1971 "A perceptual coding language for visual and auditory patterns" American Journal of Psychology 84 307-349

Lier R J van, Helm P A van der, Leeuwenberg E L J, 1994 "Integrating global and local aspects of visual occlusion" Perception $23883-903$

Richards W, Hoffman D D, 1985 "Codon constraints on closed 2D shapes" Computer Vision Graphics and Image Processing $31265-281$

Siddiqi K, Kimia B B, 1995 "Parts of visual form: computational aspects" IEEE Transactions on Pattern Analysis and Machine Intelligence 17 239-251

Wagemans J, 1995 "Detection of visual symmetries" Spatial Vision 9 9-32

Wagemans J, Lamote C, Gool L van, 1997 "Shape equivalence under perspective and projective transformations" Psychonomic Bulletin \& Review 4 248-253 